\title{
Non-communicable diseases readdressed: a review
}

\begin{abstract}
Sustainable Development Goal (SDG) 3 aims to reduce non-communicable disease (NCD) related premature mortality by 2030. NCD semerging as leading cause of death in India, its management has garnered paramount importance. This calls for an urgent integrated action plan that can foster NCD prevention by health system strengthening and multi-sectoral coordination. Despite government of India launching a number of national programmes to its effect, efforts, the approach appear to be fragmented, vertical and disease-specific, with more emphasis on rendering acute care services. Various bottlenecks like shortfall of public health facilities and poor density of health work force have been identified that potentially led to hindered NCD service delivery in the country. Such poor responsiveness of country's health system have re-emphasized on an urgent need to strengthen primary care system of India to make services more available and accessible to the most vulnerable society.
\end{abstract}

Volume 7 Issue 6 - 2018

\author{
Surekha Kishore, Surabhi Mishra \\ Department of Community and Family Medicine, All India \\ Institute of Medical Sciences (AlIMS), India
}

\author{
Correspondence: Surekha Kishore, Department of \\ Community and Family Medicine, All India Institute of Medical \\ Sciences (AllMS), Rishikesh - 249203, India, \\ Emailsurekhakeshore@rediffmail.com
}

Received: July 03, 2018 | Published: November 20, 2018

Keywords: non-communicable disease, sustainable development goal, India, national programme, primary health care

\section{Introduction}

The growing epidemic of non-communicable diseases (NCDs) in $21^{\text {st }}$ century has severely influenced national health systems, policies, and socioeconomic developments. ${ }^{1}$ Despite Millennium Development Goals (MDGs) focusing its efforts to tackle infectious diseases and child mortality, there exists a conspicuous absence of strategies pertaining to combat NCD from its framework. ${ }^{2}$ This has resulted in further widening of disparity gap between countries. ${ }^{1}$ This inadequate interpretation of MDGs in low- and middle-income countries (LMICs) has resulted in the country-specific delivery of vertical disease-specific health programs at ground level.[3] Resultantly, health system in LMICs has not only remained distorted but also ill equipped to manage this dual burden of communicable and NCDs. ${ }^{4,5}$ NCDs have emerged as the leading cause of death in India, accounting for $15 \%$ of global NCD deaths and $60 \%$ of total deaths in the country. ${ }^{6}$ It alone accounted for around $68 \%$ of global deaths in 2012; threefourth(28 million) of which are reported from LMICs. According to World Health Organization (WHO), improved control of infectious diseases, rapid urbanization, and aging population will increase projected NCD deaths to about 52 million by $2030 .^{7}$ Epidemiological evidence indicate that four major chronic diseases: cardiovascular diseases (CVDs), cancers, diabetes, and chronic respiratory diseases contributes to around $82 \%$ of all deaths due to NCDs globally. ${ }^{8}$ In India, CVD [26\%], cancer (7\%), chronic respiratory diseases (13\%), diabetes $(2 \%)$, and other NCDs $(12 \%)$ are the leading cause of NCD mortality in India. ${ }^{9}$ Findings from the Global Burden of Disease indicate that ischemic heart disease was one of the highest-ranking causes that led to premature deaths in India in 2013. ${ }^{10}$ Diabetes is predicted to affect about 109 million people between 20 and 79 years of age by $2035 .{ }^{11}$ Expanding to little more comprehensive and holistic sustainable development goals (SDGs), NCD management has garnered paramount importance as far as achievement of achieving universal health for all is concerned. ${ }^{12,13}$ SDG3 aims to reduce NCDrelated premature mortality by prevention, promotion and early treatment and management by 2030 . This calls for an urgent integrated action plan for NCD prevention through multi-sectoral coordination and health system strengthening which will lay the foundation for improved health, mitigated socioeconomic impacts, and accelerated sustainable development.

Presently, India represents third largest economy and one of the most populous countries in the world. Despite significant improvements in health care indicators in the past decade, Indian health-care system continues to contribute disproportionately to the global disease burden. ${ }^{14}$ India is currently experiencing a phase of rapid health transition, wherein mounting magnitude of NCDs is gaining prominence with substantial adverse impact on health and economic productivity. ${ }^{15}$ Therefore, it is important to perceive India's health system preparedness toward meeting growing NCD challenge. Recognizing NCD's growing threat in India, the Ministry of Health and Family Welfare (MoHFW), government of India (GoI) has launched National Programme for Prevention and Control of Cancer, Diabetes, CVD and Stroke (NPCDCS), a national program for control of NCDs. NPCDCS seeks to integrate NCD interventions into the framework of public-health delivery through the promotion of healthy lifestyle habits, early diagnosis of diabetes, hypertension, CVDs and stroke, the establishment of NCD clinics at CHCs, capacity building for health promotion, regular supervision, monitoring, and evaluation of the programme. Additionally, preventive, curative and rehabilitative care for the elderly in various government health facilities is envisaged under the National Programme for Care of the Elderly. The National Tobacco Control Programme has facilitated the implementation of the tobacco control laws in the country. The launch of the National Mental Health Program ensured the availability and accessibility of appropriate mental health care for the vulnerable population. Despite commendable efforts, the programme appear to be fragmented, vertical and disease-specific, with more emphasis on rendering acute care services. There remains considerable inadequacies in NCD care delivery services at both primary and secondary care level. The NCD Scorecard (http://www.ncdglobalscorecard.org), developed from United Nations political declaration after High-level Meeting on the Prevention and Control of NCD in 2011 indicates India's very low progress in terms of health system response for NCD and low performance in its efforts to reduce risk factors and conducting disease surveillance of disease. India's present health system readiness is 
an issue of concern. Some of the major bottlenecks in the smooth delivery of NCD services are: shortfall of public health facilities; non-availability of appropriate infrastructure; poor density of health workforce trained in NCD-care delivery services; discordance in drug availability; absence of focus on NCDs in IPHS standards. As per MoHFW Rural Health Statistics 2014-2015, the number of subcentres, PHCs, and CHCs have increased over the last decade. The current numbers are yet not sufficient to meet their population norms. There is yet a shortfall of $20 \%$ in SCs, $22 \%$ in PHCs, and $32 \%$ in CHCs in India. ${ }^{6}$ There is a significant gap in infrastructure such as laboratory, Operation Theater, beds, at primary care level, on the basis of number of health facilities functioning in the country. Only $20.72 \%$ PHCs are functioning as per IPHS norms. ${ }^{16}$ Shortage of skilled health workers, trained in providing chronic at field level is another critical issue. In PHC, there is a shortfall of $49.2 \%$ for female health assistant and $61.3 \%$ for male health assistants. $10.5 \%$ of the sanctioned posts of HW (Female)/auxiliary nurse midwives (ANMs) were vacant at SC and PHCs, $27.0 \%$ of the sanctioned posts for doctors at PHC were vacant. The statistics highlight that $8.1 \%$ of the PHCs were functioning without a doctor, $38.1 \%$ without a laboratory technician and $21.9 \%$ without a pharmacist. ${ }^{16}$ India depicts a disappointing picture in terms of density of health workforce per 10,000 populations. Compared to global scenario, only 7 physicians and 17.1 nursing/midwife personnel are available per 10,000 population in India. ${ }^{17}$ The availability of essential technologies and medicines to treat NCDs at the primary care level is an essential prerequisite. While there is an essential drug list for all PHCs, there remains a discordance in the availability of these recommended and essential drugs for NCDs, which are either not available or their supplies are intermittent. While the SDGs aspire to achieve universal health coverage, India must strive toward improving efforts to tackle NCD management and emphasizes on the relevance of empowering the primary care system. It is critical that NCD care service delivery models at the primary care level are articulated well keeping in view the Indian scenario and implemented so as to make them available and accessible to the most vulnerable sections of society.

\section{Acknowledgements}

None.

\section{Conflict of interest}

The author has no conflicts of interests in this work.

\section{References}

1. WHO. Global Status Report on Noncommunicable diseases 2010.

2. Fehling M, Nelson BD, Venkatapuram S. Limitations of the millennium development goals: A literature review. Glob Public Health. 2013;8:11091122 .

3. The NCD Alliance. NCD Alliance Position Paper: Post-2015 Global Health Thematic Consultation.

4. The NCD Alliance. European Commission Public Consultation: Towards a Post-2015 Development Framework.

5. Lopez AD, Williams TN, Levin A, et al. Remembering the forgotten noncommunicable diseases. BMC Med. 2014;12:200.

6. World Health Organization. Burden of NCDs and their Risk Factors in India.

7. World Health Organization. Global Status Report on Noncommunicable diseases 2014.

8. World Health Organization. Noncommunicable Diseases.

9. World Health Organization. Noncommunicable Diseases Country Profiles 2014.

10. World Health Organization. Global Burden of Disease Data.

11. Yesudian CA, Grepstad M, Visintin E, et al. The economic burden of diabetes in India: A review of the literature. Global Health. 2014;10:80.

12. World Health Organization. Health in 2015: from MDGs, Millennium Development Goals to SDGs, Sustainable Development Goals.

13. Minas H, Tsutsumi A, Izutsu T, et al. Comprehensive SDG goal and targets for non-communicable diseases and mental health. Int J Ment Health Syst. 2015;9:12.

14. Patel V, Parikh R, Nandraj S, et al. Assuring health coverage for all in India. Lancet. 2015;386:2422-2435.

15. Yadav S, Arokiasamy P. Understanding epidemiological transition in India. Glob Health Action. 2014;7:23248.

16. Rural Health Statistics, Ministry of Health and Family Welfare Statistics Division, Government of India.

17. World Health Organization. World Health Statistics 2015. 